\title{
LOS ERRORES DE KANT. LA CRÍTICA DE ROSMINI AL IDEALISMO TRASCENDENTAL
}

\author{
Kant's Errors. Rosmini's Critique of Kant's Transcendental Idealism
}

Jorge Alfonso Vargas*

Resumen

El artículo analiza la crítica de Antonio Rosmini a la filosofía de Kant. Se postula a la crítica a Kant como una cuestión de principios que critica al subjetivismo kantiano y la equivocada formulación del problema ideológico (del origen de las ideas) en relación a los juicios sintéticos a priori. Se concluye que Kant confunde los predicados con los atributos, los conceptos con las cosas, y lleva su filosofía al subjetivismo.

Palabras clave: Ser, sentimiento, conocimiento.

Abstract

The article analyses Rosmini's critique of Kant's philosophy. The author aludes to the criticism of Kant as a question of principles that criticizes Kant's subjectivism and the wrong formulation of the ideological problem (of the origin of ideas) related to the synthetic a priori judgments. Ending by holding that Kant confuses predicates with attributes, concepts with things, and leads his philosophy to subjectivism.

Key words: Being, feeling, knowledge.

La filosofía de Antonio Rosmini ${ }^{1}$ está despertando cada vez más interés en quienes tenemos la suerte de haber llegado a conocerla. Sorprende la claridad, amplitud y sistematicidad de su obra. Atrae la simplicidad de su principio único, la idea del ser, y de su experiencia fundamental, la del sentir y el entender. Sorprende la aguda crítica de Rosmini a la tradición, en la persona de sus pensadores más destacados, como también llama la atención la forma como realiza una crítica histórica la que en vez de dejar de lado a alguna doctrina o a un pensador, los sobrepasa e integra a su sistema, de manera tal que uno se pregunta qué habría sido de la filosofía moderna si la obra hubiese sido más conocida.

\footnotetext{
${ }^{1}$ Este estudio se basa en la obra Nuovo saggio sull'origine delle idee de Antonio Rosmini en la versión inglesa A New Essay concerning the Origin of Ideas Vol. 1, 2, 3. Durham: Rosmini House, 2001. (Las traducciones son del autor). Una buena introducción es la obra de Humberto Muratore. Antonio Rosmini. Vida y pensamiento (Madrid: BAC, 1998). Cfr. Jorge Alfonso. "El ser como principio: Unidad y Sistematicidad en la Filosofía de Antonio Rosmini". Pensamiento, Vol. 64, N ${ }^{\circ}$ 240. Madrid: Universidad de Comillas, 2008:251-266. Agradezco al Dr. Juan Francisco Franck de la Universidad Católica de Argentina (UCA) la revisión de este artículo y sus atinados consejos.
} 
Entre las críticas, la realizada a Immanuel Kant sobresale, sobre todo, por el lugar que el filósofo alemán ocupa en la historia de la filosofía moderna $^{2}$ y por la centralidad que su crítica tiene para la comprensión de la propia filosofía de Rosmini (López-Riobóo, 1977). Por eso queremos darla a conocer empezando por los principios de la filosofía rosminiana para luego entrar en materia y analizar los errores que Rosmini encuentra en Kant, a quien admira por muchas razones, pero al que no puede seguir, por otras derivadas de sus propios principios.

Rosmini puede decir, con toda propiedad, que la idea del ser es la luz del alma. El alma intelige porque no necesita tener todas las ideas sino solamente una, la del ser. La idea del ser es el inicio de todo conocimiento. ${ }^{3}$ Si somos una tabula rasa antes de conocer el mundo es porque tenemos la idea del ser indeterminado todavía, pero, aun así, estamos abiertos a él, a todas sus posibilidades. ${ }^{4}$ En consecuencia: en primer lugar, el ser es el elemento que entra en todas nuestras ideas y, en segundo lugar, el ser es lo que queda como principio último de nuestras ideas después de todas las abstracciones posibles. La última abstracción es la del ser. La última, pero no la menos importante, puesto que sin ella no podemos pensar nada, ninguna idea es posible. Por lo tanto, o decidimos no pensar — cosa imposible - o si pensamos, pensamos el ser. No podemos negar el ser porque sería negar el pensar. Por eso mismo, el proceso cognoscitivo asciende desde los pensamientos acerca de los cuerpos - cuyas propiedades corporales refleja la imaginación - hasta el conocimiento de los entes espirituales que tienen todo lo requerido para la subsistencia pero que no ofrecen base para ninguna imagen sensible y cuyo término es un objeto puro del pensamiento.

Rosmini reconoce que el mayor logro de Kant es haber visto, con mayor perspicacia que otros pensadores, la diferencia esencial entre las dos operaciones del espíritu: el sentir y el entender. Así, según Rosmini, lo que Kant entendió bien fue que el entendimiento es activo y el sentido pasivo; que los sentidos aportan la materia y el intelecto la forma y que las intuiciones dependen de los sentidos; en cambio, los conceptos dependen de las

\footnotetext{
${ }^{2}$ Para efectos de la filosofía de Immanuel Kant remitimos a su Crítica de la razón pura. Buenos Aires: Losada, 1961.

${ }^{3}$ Entre idealidad e inicialidad hay una relación dialéctica: es la idea del ser la que da inicio al conocimiento, la idea, no el concepto. Para entender a Rosmini hay que evitar la tendencia moderna a confundir las ideas con los conceptos. Cfr. Pier Paolo Otonello, 1989.

${ }^{4} \mathrm{El}$ ser se refiere al ser ideal que es divino pero no es Dios (Dios es persona). Hemos preferido no introducir en este artículo el tema de Dios para fijar nuestra atención en el argumento puramente filosófico, pero en un contexto general de la filosofía rosminiana es esencial comprender el lugar de Dios en su sistema. Para este propósito recomendamos, Giorgio Giannini (1994:9-24) y Pier Paolo Otonello (1998:99-113).
} 
funciones. Sin embargo, sostiene Rosmini, Kant se equivocó al hacer de los objetos del pensamiento algo subjetivo. ${ }^{5} \mathrm{Y}$ el problema (ideológico, lo llama Rosmini) reside en el rechazo de Kant de la creencia en la causalidad como un hecho, ya que uno tendría que creer en ella para aceptar que los entes se nos presentan efectivamente como son ¿Por qué Kant se equivoca? Porque al no aceptar el principio de causalidad como algo externo, se ve inclinado a pensar que viene del interior, del espíritu, que es casi una creación de éste. Por eso, Rosmini al recordar el pensamiento de Kant advierte que este filósofo no encuentra nada a medio camino entre los sentidos y el intelecto para explicar el conocimiento. Crea, así, lo que Rosmini llama el problema del puente entre lo interior y lo exterior, cuando en verdad para él no hay tal problema. Lo que Kant hace, según Rosmini, es separar los sentidos del intelecto y, así, al separar el intelecto de los sentidos pierde la conexión con la realidad. De ahí, la importancia de la causalidad que nos hace presente los entes en la medida de que nos afectan.

Es la incomprensión de la causalidad lo que hace que el argumento de Kant se debilite, según Rosmini. Detengámonos en el tema de la causalidad, de acuerdo a la visión rosminiana, para luego volver a nuestro tema. Cuando un nuevo suceso ocurre, algo nuevo comienza a existir. La existencia es una operación lo que implica un operador, porque una sustancia es un ente con capacidad para operar, de ser una fuerza. Esta es la definición de sustancia que llama la atención por estar más cerca del origen, más cerca de la energeia aristotélica, como destaca Heidegger y, por eso, ponemos como el primer desacuerdo entre Rosmini y Kant, ya que este naturalismo espiritualista no se aviene muy bien con el idealismo intelectualista de Kant, ${ }^{6}$ por eso sostiene que "La existencia misma es una operación (un acto). Cuando la existencia de una

\footnotetext{
${ }^{5}$ Ciertamente que Kant no se ve a sí mismo como un subjetivista, puesto que lo que más le preocupa es justamente desentrañar en qué consiste la objetividad de la razón. Cfr. Ernst Cassirer (1993); Lo que sucede es que Rosmini va más allá de la razón porque lo que le preocupa es el fundamento anterior, lo que hace posible el conocer, lo que nos vuelve sujetos inteligentes. Cfr. Michele Federico Sciacca (2003).

${ }^{6}$ Este naturalismo rosminiano es, a su vez, metafísico, ya que este mismo tema de la causalidad supone una explicación en estos términos dado que no es un tema trascendental sino trascendente. Al respecto, vale la pena traer a colación la siguiente afirmación de G. Giannini: "La diferencia entre trascendente y trascendental consiste en que mientras lo trascendente es objetivo, lo trascendental es subjetivo: el primero capta lo que es (el noúmeno), el segundo lo que aparece (el fenómeno). A la objeción kantiana: el conocimiento de lo trascendente es contradictorio, en cuanto nos daría la experiencia de aquello de lo cual no se tiene experiencia, se responde que la experiencia de lo trascendente no es inmediata, sino mediata, y la experiencia mediata es aquella por medio de la cual se va del efecto a la causa. La experiencia del tren que marcha es inmediata; la experiencia de la energía que la hace marchar es mediata; pero sin ésta no sería posible aquella" (1997:96).
} 
cosa comienza - y yo considero así esta existencia como una operaciónnecesariamente afirmo alguna existencia primera, anterior, a la cosa. Esta existencia es, precisamente, lo que llamamos causa" (2001:306). Ésta es una afirmación importante en Rosmini. Podríamos preguntarnos sobre su status epistémico ¿Ontológico, epistemológico, nominal? Para Rosmini, el concepto de lo que pasa contiene ya el concepto de causa, por lo mismo que causa y efecto son términos complementarios. No es ni siquiera un juicio sintético a priori (todo efecto tiene una causa), ya que no se trata de que el concepto del predicado (causa) esté contenido en el concepto del sujeto (efecto). Para Rosmini, se trata de una afirmación ontológica, no metafísica aún. ${ }^{7}$ Pero sigamos el argumento de Rosmini. El problema es justamente el problema ideológico, el del origen de las ideas, del que habla Rosmini y que Kant no habría resuelto bien ¿Cómo podemos concebir el pasaje de una cosa de la noexistencia a la existencia? Si nos limitamos al plano sensorial, no hay ningún problema. Existe lo que vemos, tocamos o, en general, sentimos, y que antes, justamente, no veíamos, tocábamos o sentíamos. Mas, si sólo tuviéramos sensaciones sin el poder de concebir algo como existiendo, distinto de nosotros mismos, no podríamos nunca concebir el pasaje de la no existencia a la existencia, lo que lleva al siguiente problema ¿Cómo percibimos los entes dotados de existencia? ¿Cuál es el origen de las ideas? Éste es el problema ideológico. El comienzo de las ideas está en los entes que impresionan los sentidos, pero éste es sólo el comienzo, ya que pensar requiere ascender del sentir al inteligir y al razonar, en este orden. ${ }^{8}$ Sin embargo, todo comienza en las cosas y en su poder de causar impresiones sensoriales. Por eso, la creencia en la causalidad es esencial al proyecto de Rosmini.

De las muchas formulaciones del tema, escogemos una que se refiere a lo ya planteado mediante el análisis de la afirmación todo efecto tiene una causa. Para Rosmini, este no es un juicio sintético a priori y, ahora lo podemos decir con mayor seguridad, es un hecho ¿Por qué? Porque según Rosmini, la proposición no expresa un juicio a priori sino la aplicación de un juicio a priori. Y la aplicación de un juicio a priori es un hecho, no es un principio. El orden del argumento es el siguiente: $1^{\circ}$ Un principio a priori: todo efecto debe tener una causa; $2^{\circ}$ Un hecho general: todo evento es un efecto; $3^{\circ}$ La aplicación de un principio general: Cada cosa que pasa debe

\footnotetext{
${ }^{7}$ Decimos no metafísica aún porque es, justamente, la imposibilidad de que la realidad sea el origen de las ideas debido a la propia naturaleza de éstas (universales, abstractas, infinitas), lo que va a requerir una solución metafísica a lo que hasta ahora ha sido una descripción puramente ontológica.

8 “(...) la gradación que se determina mediante la intuición del ser, es ésta: sensiente, inteligente, intuente, intelectivo. La intuición del ser vuelve inteligente al sensiente, intuente al inteligente e intelectivo al intuente", Giannini (1997:71).
} 
tener su causa. El problema no está en el primer nivel. Todos vemos, sentimos, algo que no es nuestro cuerpo, que es extra-subjetivo y nos damos cuenta que existe porque nos afecta; tampoco el problema está en el tercer nivel puesto que luego de concebir el evento como un efecto suponemos que tiene una causa. El problema verdadero está en el segundo nivel, el de pensar un evento bajo el concepto de efecto. Es este ir de los efectos a las causas extrasubjetivas lo que comienza a separar a Rosmini de Kant porque éste le da a las causas el carácter a priori de categorías de la percepción pero no el de una imposición factual, como lo hace Rosmini y, así, la filosofía se desliza hacia el subjetivismo, según Rosmini, a pesar del esfuerzo de Kant por no caer en él.

Para Rosmini, el problema del subjetivismo kantiano reside en que Kant no puede ver que el ser objetivo y el ser subjetivo son el mismo ser. Éste es un punto central en Rosmini. Pero es mejor dejarlo hablar a él mismo para poder seguir su argumento. "El supuesto sobre el cual Kant construye su sistema y del cual no ofrece ni la más mínima evidencia... se debió a su incapacidad para advertir que el ser tiene dos modos (uno subjetivo, el otro objetivo) y que en ambos es idéntico. Ser en el modo objetivo es el ser que se hace conocido, y se hace conocido como es, aun cuando es subjetivo. Pero puesto que el ser es idéntico (en ambos modos), el conocimiento es eficaz y verdadero" (2001:331). Lo que hace difícil ver como idénticos el ser real y el ser posible es el problema de cómo se puede pasar del ser posible al ser real cuando en verdad para Rosmini no hay, por decirlo así, traslación de lo posible a lo real. Lo que sucede, según entiende Rosmini, es que el tema ha sido enfocado desde la perspectiva puramente gnoseológica cuando la solución a esta dificultad ideológica es para él metafísica: hay un único principio que hace que el sentir sea lo mismo que el entender, y así — según ejemplo de Manzoni que Sciacca repite- el trigo en la mente del agricultor y el trigo que éste cosecha es el mismo trigo bajo dos formas diferentes o modalidades (Sciacca, 1957:265).

Siendo el ser idéntico en los dos modos, decir que el ser es subjetivo está mal dicho puesto que es una reducción de lo extra-subjetivo a lo subjetivo; además, la existencia extrasubjetiva es reducida en su status ontológico al hacer de ella sólo una existencia subjetiva, como lo hace Kant. La existencia objetiva es cierto que no proviene de los sentidos —en eso está de acuerdo con Kant- pero, si bien la existencia proviene de los sentidos, no es conocida por su inteligibilidad, por su pensabilidad, que comienza, agregaríamos nosotros, por ser experimentada como un ser en sí, no como una mera sensación o un estado psicológico. Sin esta inteligibilidad añadida nada sería conocido sino que meramente sentido ¿Cómo sucede esto? En primer término, mediante la atribución de universalidad a la sensación particular; atribución posible por la idea del ser que permite que lo que sentimos 
corresponda a un ser que no se confunde con nuestras sensaciones o estados. No es que le atribuyamos a las cosas algo nuestro, como parece sugerir Kant —decimos "parece sugerir" porque tenemos dudas sobre si Kant realmente lo pensó así, o si son sus intérpretes los que han hecho de él un subjetivista— sino que lo que sucede es que, en palabras de Rosmini, nosotros reconocemos esta universalidad; no la creamos, no la sacamos de nuestro interior. ${ }^{9}$ En la formulación de Rosmini cuando enunciamos el juicio tal y cual entidad real existe aplicamos el predicado universal de existencia a un sujeto particular. Mas, de esto no se sigue que con nuestra actividad ponemos la existencia universal en la cosa percibida. Sólo la encontramos en su particular existencia y así nos referimos a su existencia universal.

No es, entonces, que al conocer ponemos en la cosa lo que antes estaba en nuestro intelecto. Lo que sucede es que, como el ser subjetivo es el mismo que el ser objetivo, percibimos en las cosas lo que está en ellas; la existencia objetiva se conoce por su existencia subjetiva; pero no se reduce a ella, la existencia subjetiva reconoce sólo lo que pertenece objetivamente a las cosas. El error de Kant residiría en no distinguir el concepto de la cosa, o reducir la cosa al concepto. Este error consiste en no ver que el concepto es siempre universal y la cosa es siempre particular, lo que lleva a Kant a equivocarse al respecto al considerar - según Rosmini- que el concepto intelectual y la cosa correspondiente a él eran una misma entidad (2001:332) y que todo el universo era el producto del entendimiento humano y de la sensibilidad, en definitiva, del hombre. Cuando, de acuerdo a Rosmini: "Él debió haberse dado cuenta que la parte en que el intelecto contribuye al conocimiento está confinada a hacer conocido lo que la cosa contiene sin contribuir en nada a ella. Él habría hecho esto si hubiera considerado cómo las formas objetivas, que están en la mente, se restringen ellas mismas a la medida de las cosas reales, subjetivas y sensibles" (2001:332) ¿Por qué sucede esto? Porque Kant no entiende — según Rosmini- la naturaleza de la percepción intelectual. La percepción intelectual es la visión de la relación entre una idea (la existencia) y lo que es percibido por los sentidos (una sensación). De acuerdo a la crítica de Rosmini, Kant ve las ideas universales tan estrechamente mezcladas con el objeto percibido que cree que ellas juntas conforman el objeto, sin distinguir - y éste es un punto fuerte en Rosmini- entre el predicado y el atributo.

\footnotetext{
9 Al parecer en la crítica de Rosmini habría un punto no muy claro en su discusión. Kant rechazó las ideas innatas justamente como subjetivas. Su idea de lo a priori no es lo innato sino lo adquirido, pero lo adquirido no son ideas sino leyes del conocimiento. Querer derivar la universalidad de estas leyes de la experiencia es imposible (ex pumice aquam). En esto, Kant va bien encaminado pero su solución gnoseológica no parece tan apropiada como la metafísica de Rosmini. Cfr. J. F. Franck (2007:53-76).
} 
Si se trata de distinguir, entonces, entre los predicados y los atributos, el error de Kant está en confundir los atributos de las cosas con los predicados lógicos, el confundir los conceptos con las cosas y el no poder ver la diferencia, al punto que el problema del origen de las ideas se plantea en términos puramente lógicos ¿Cómo son posibles los juicios sintéticos $a$ priori? Lo que nos lleva a la crítica de Rosmini respecto a formular el problema. La formulación clásica parte de la distinción entre los juicios analíticos, aquellos que el predicado está contenido en el sujeto y que pueden demostrarse como verdaderos simplemente mediante un análisis del sujeto; de los juicios sintéticos en que el predicado no está contenido en el sujeto, juicios que sólo sabremos si son verdaderos a posteriori, luego de su comprobación empírica. Los primeros son siempre verdaderos pero obvios; no muy informativos diríamos hoy en día, ya que no aprendemos nada nuevo de ellos; por otra parte, con los segundos aprendemos algo nuevo - pero no universal- y luego de una comprobación empírica a posteriori. Lo importante para la ciencia es la posibilidad de un tercer tipo de juicio que sea un juicio sintético, que agregue algo nuevo al conocimiento y, que sea, a la vez, a priori, que sea verdadero por el mero análisis de los conceptos, sin recurrir a la experiencia. Estos juicios para Kant son posibles y son, básicamente, los juicios de las matemáticas o de la geometría: $3+2=5$, los ángulos interiores de un triángulo son igual a 90 grados, etc.

Kant ha percibido, claramente, que cada función de nuestro entendimiento se reduce, en último lugar, a un juicio y que para poder enjuiciar hay que tener con qué enjuiciar: hay que tener ciertas nociones o conceptos puesto que juzgar, en el campo epistemológico, significa someter lo particular a lo universal. De forma tal que el problema consiste en explicar estos conceptos anticipados, es decir, aquellos presupuestos antes de las sensaciones; y en esto está de acuerdo el mismo Rosmini. Pero ¿es verdad que hacemos estos juicios sintéticos a priori? Rosmini piensa que no, y precisa lo siguiente: "Kant sostiene que hacemos ciertos juicios sintéticos a priori (...). Para entender lo que voy a decir nótese cuidadosamente — advierte-- cuál juicio sintético a priori yo niego. Estos son juicios en los cuales se hace el intento de añadir, a un sujeto presupuesto, un predicado que no está ni contenido en el concepto formado por la mente, ni es proporcionado por la experiencia sensible". Siguiendo a Rosmini podemos advertir que aquí comienza la crítica del subjetivismo kantiano, ya que si en un juicio sintético a priori le añadimos algo al sujeto, que no estaba en el sujeto, ni proviene de los sentidos ¿De dónde podría venir si no del espíritu, es decir, del mismo sujeto que crea esta nueva realidad; así, el 5, que no estaba ni en el 3 ni en el 2, de dónde podría venir entonces? Rosmini manifiesta que el lenguaje de Kant no es el apropiado para describir estas funciones del espíritu; incluso, cree que este 
lenguaje es demasiado material para describir algo espiritual. El punto es muy importante y merece atención especial.

Examinemos lo que Rosmini tiene que decir respecto de lo que llama el tema del juicio primitivo, aquel presente en el conocimiento de cualquier realidad y que nos permitirá entrar en el tema kantiano aunque aquí comienzan a manifestarse, también, las diferencias principiales. Este tipo de juicios es definido de la siguiente manera: “(...) el juicio primitivo de nuestro espíritu, aquel juicio mediante el cual nace la percepción intelectual, yo lo llamo sintético y a priori porque se forma una unión espiritual con una cosa dada por lo sentidos, que se convierte en sujeto, y otra que no entra en el sujeto en cuanto dato de los sentidos pero que se encuentra sólo en el intelecto y el predicado" (2001:359). Sólo en este sentido Rosmini está de acuerdo con Kant. Los desacuerdos comienzan con un análisis detallado del lenguaje kantiano respecto a este tema. Por ejemplo: "Decir —advierte Rosmini- 'El predicado no existe en el concepto del sujeto es enteramente diferente de decir': El predicado no existe en el sujeto. La primera es la frase de Kant que contiene la ambigüedad y el error; la segunda es la única que acepto y reconozco como exacta" (2001:360). La diferencia más marcada reside en el cuidadoso deslinde de la expresión en el sujeto ¿Se trata de algo que está dentro del sujeto como el agua en la jarra o de algo que está en el concepto del sujeto como una de sus notas constitutivas? Kant parece referirse siempre al concepto del sujeto, lo que Rosmini le censura porque, para él, el sujeto que está dado por los sentidos no merece todavía el nombre de sujeto en propiedad; porque sólo ha sido sentido y no pensado, lo cual lleva a Rosmini a una de sus precisiones más agudas y que debemos escuchar con atención: "En una palabra: los sujetos de nuestros juicios son o proporcionados sólo por nuestros sentidos o ya son concebidos por nuestro intelecto. En el segundo caso, tenemos el concepto del sujeto de nuestro juicio; en el primer caso tenemos de alguna manera el sujeto del juicio, el sujeto en potencia, que se convertirá en sujeto cuando el juicio se haya hecho, pero no poseemos su concepto. Sólo cuando añadimos el predicado al sujeto y formamos un juicio, en realidad, adquirimos, finalmente, a través de este mero juicio, el concepto del sujeto" (2001:360). El punto fuerte de Rosmini está, en términos breves, en que, tal como Kant lo sostiene, el conocer depende de los sentidos y del intelecto, pero lo que se dice de un sujeto y que no proviene de los sentidos tampoco proviene del sujeto que lo dice, no es una creación del espíritu como sugiere Kant sino, más bien, es un descubrimiento del espíritu que, aplicando la sola idea del ser va construyendo realidad sobre la base de distinguir lo que siente de lo que sabe y reconociendo que lo que sabe, lo sabe porque se le ha presentado objetivamente y, por lo mismo, se puede, si se quiere, comprobar ya sea en los hechos, o simplemente en el asentimiento de los espíritus. Pero, 
para pasar de estas cuestiones de principios a uno de aplicación de estos principios debemos detenernos algo más en algunos ejemplos clásicos de juicios sintéticos a priori para entender en la práctica las objeciones de Rosmini a Kant. Uno de los ejemplos clásicos es la suma $7+5=12$ que sería un juicio sintético porque añade algo nuevo que no está en el 7 ni en el 5 y sería a priori porque no necesita de la experiencia para comprobar su verdad: ¿El 12 no está en el concepto de 7, o de 5, o en el de su suma? Rosmini no cree que éste sea un juicio sintético sino analítico, pero en un sentido diferente al de Kant. Vamos por parte, y prestemos atención a sus argumentos. Kant afirma que el concepto de 12 no puede derivarse de la suma de $7+5$, sino que es algo nuevo, un producto del espíritu. Nada hay en la expresión de esta suma que permita esta deducción salvo que uno se ayude con los dedos para verificarla, señal externa que demuestra la verdad de la afirmación. Sin embargo, para Rosmini esta explicación es irrelevante, ya que "El necesitar nosotros de alguna señal externa para derivar el número 12 de $7+5$ no prueba que el concepto de 12 no esté comprendido en el concepto de la suma de los dos números. Por el contrario, prueba que si no lo estuviera seríamos incapaces de extraerlo aun con la ayuda de señales que no añaden nada al concepto sino, simplemente, nos ayudan a reconocer la misma cosa bajo dos formas diferentes o expresiones" (2001:346). Advierte muy bien Rosmini que la forma de un concepto es una cosa y el concepto otra, ya que podríamos necesitar recurrir a los sentidos y ver que 7 bolitas de cristal más 5 bolitas de cristal son 12 bolitas de cristal, o podemos meramente pensarlo; sin embargo - Rosmini parece estar en lo cierto- el concepto de 12 tiene que estar antes en la suma de $7+5$ para poder deducirlo de ella. Es en este sentido que el juicio es analítico y sintético a la vez para Rosmini: No hemos puesto nada en esta suma que no esté ya en ella sólo; hemos descubierto una relación entre el número 7 y el número 5 que seguramente a un niño sorprende como novedoso pero que no es tal. Por lo demás, a veces, lo que vemos como distinto lo es por su diferente forma cognitiva: así el lucero de la tarde y Venus son una misma cosa bajo distintos nombres, por poner un ejemplo. Y aquí está el error de Kant, quien vería en la suma un juicio sintético a priori sin darse cuenta que en el concepto de un número ya hay una síntesis que ha encontrado la unidad en un colectivo porque ya estaba en él, no porque el espíritu del observador lo haya puesto ahí. Rosmini advierte que en la naturaleza no hay colectivos sino individuos; por eso, cualquier concepto numérico ya implica algo que está por sobre lo que encontramos en la naturaleza, en este caso en el concepto de colectivo, lo que sucede es que la mente contribuye con la idea de unidad mediante la cual unifica individuos y los convierte en un colectivo. Por consecuencia, en toda cantidad hay implícito un juicio sintético a priori. El error de Kant estaría en buscar el juicio sintético en la suma de 7+5 en vez de 
Jorge Alfonso Vargas

buscarlo y encontrarlo en el concepto de 7 y de 5 o de cualquier otro número (2001:129).

¿De donde viene el error? En primer lugar, de la mala comprensión del concepto de unión, de síntesis. Volvamos al tema con mayor precisión. Rosmini llama la atención sobre el hecho que síntesis significa unión y que el sentido que Kant le da es un sentido metafórico. Por cierto, no se trata de una unión física. Justamente éste el problema, cómo explicar la unión entre sensaciones e ideas lo que no es ciertamente una cuestión física ya que como sostiene Rosmini: "Cuando yo digo: Yo uno un predicado con un sujeto, puedo entender que inserto este predicado en el sujeto como pongo una piedra preciosa en un anillo, o como coloco una tabla de madera en la casa que estoy construyendo. La piedra y la tabla están en el anillo o en la casa porque yo las he puesto allí. Tal es el sentido en el que Kant lo entiende" (2001:356). Y éste es el sentido que Rosmini rechaza, por varias razones. La principal, porque en este caso no estamos hablando en un sentido físico sino espiritual y es porque hablamos metafóricamente; hablan del unión pero no como en el caso de la piedra en el anillo o la tabla en la casa. El problema está, justamente, en que estamos tratando de entender un fenómeno espiritual mediante una metáfora física. No consiste la palabra síntesis o unión en añadir nuestra idea de existencia a un ente, sino, simplemente, en concebir la relación entre éste y nuestra noción de existencia por medio de la unidad que aporta nuestro espíritu. Captar una relación no significa confundir o mezclar los dos términos de la relación en una sola cosa. Ésta sería una especie material de unión. Sería como si dos líquidos fueran vertidos en un recipiente o dos ingredientes mezclados en una comida. Por el contrario, cuando concebimos una relación los dos términos que relacionamos son mantenidos apartes y son unidos sólo por un acto del espíritu que considera uno en relación al otro y, consecuentemente, el espíritu encuentra una relación entre ellos. Pero esta relación no está en el sujeto que realiza este juicio, sino que es descubierta en las cosas y se tiene que atener a la naturaleza de las cosas y, por lo mismo, hace posible el conocimiento. Pero sigamos examinando los argumentos en contra de la formulación kantiana de los juicios que hacen posible un conocimiento nuevo. Para una mejor comprensión de la crítica de Rosmini a Kant es importante analizar otro ejemplo: el del atributo de cantidad aplicado a cualquier ente. De nuevo, es necesario admitir, siguiendo a Rosmini — como lo hemos hecho ya- que para percibir algo, en este caso la cantidad no necesitamos creer, como Kant, que hemos puesto en la casa algo que proviene de nuestra mente, la cantidad, y que por eso podemos decir la casa es grande. El error está en creer que al percibir una casa le añadimos una idea, en este caso la de cantidad, que sacamos de nuestra mente, cuando en verdad el atributo lo descubrimos en la casa real y particular. Es verdad que el juicio — que en este caso hacemos- 
descubre la relación entre la idea universal de cantidad y la casa en particular de la cual hablamos. Esto lo concede Rosmini a Kant, pero lo que no le concede es que se necesite sacar de la mente todas las ideas para poder percibir las cosas cuando, en verdad, la mente va descubriendo la realidad en la medida que se aplica a ello. No es, entonces, que la cantidad real y la ideal sean la misma cosa, o más bien que no exista la cantidad ideal como quiere Hobbes, o que exista sólo idealmente como lo pretende Berkeley. Al ser humano que siente e intelige le basta distinguir lo que siente de lo que es para ir descubriendo los atributos de las cosas que posteriormente va ir articulando en enunciados lógicos. El tema es escurridizo, sin embargo, y el problema kantiano sigue penando ¿De dónde vienen las ideas si no vienen del exterior? Advierte Rosmini que será siempre necesario distinguir entre la idea y la cualidad particular en una cosa. La idea es la regla de nuestros juicios; la cualidad particular en la cosa externa, que reconocemos, es el resultado de nuestro juicio; es lo que hemos llegado a conocer por medio de ese juicio. No es cierto, entonces, que nuestro intelecto inserte la idea en la cosa, aunque use la idea para conocer lo que está en la cosa sentida. Lo que sucede, realmente, es que nuestro espíritu coloca lo sentido bajo la Idea (del ser) y, así, hace de la cosa real externa un verdadero objeto de cognición. Según J. F. Franck, Rosmini universaliza la sensación mediante una operación racional, no ya intelectiva, que realiza la unión de lo sensible con lo inteligible y de lo inteligible con lo racional, pero que no necesita de todas las ideas previas para luego colocarlas en las cosas sino que, más bien, nuestro espíritu va descubriendo poco a poco las relaciones entre las cosas (2007:205-256).

Queda claro, entonces, que en el problema de la síntesis se trata de una descripción de un fenómeno espiritual y que se trata, más bien, de iluminar el espíritu descubriendo relaciones, no creándolas. Se trata de hablar y de pensar en los atributos de las cosas; aunque debemos tener cuidado en no confundir los predicados con los atributos. En este sentido, los predicados, que no provienen de los sentidos ni de la experiencia, están en el sujeto, pero están ahí porque los hemos encontrado en las cosas. Rosmini puede decir que un predicado está en el concepto de un sujeto una vez formado el concepto. No es que el sujeto que habla haya puesto en el objeto de su afirmación lo que no podía venir de los sentidos; sino que, en verdad, lo que hace es descubrir con la luz del espíritu y la simple y única idea del ser los atributos de las cosas. Rosmini no olvida nunca que la sensación es una forma de comunicación con el exterior, con las cosas, mientras que Kant, según él, sacrifica las cosas a los conceptos. Quizá la crítica sea —o aparezca— demasiado dura porque el problema sigue molestándonos ¿Cómo podemos distinguir el tamaño ideal de una casa del tamaño real de ésta? La diferencia parece de sentido común pero el problema no se resuelve así de fácil. El problema está justamente en la 
forma de concebir cualquiera de las cuatro categorías, cantidad, cualidad, relación y modalidad. Para Rosmini "El error de Kant aquí consiste en asumir que las cuatro categorías, cantidad, cualidad, relación y modalidad, son condiciones de la percepción intelectual o, como él dice, de la experiencia. De hecho, ellas son meramente condiciones de la existencia de las cosas exteriores” (2001:335) ¡No podría haber quedado más clara la diferencia que separa a ambos pensadores! Si basta la idea de existencia para percibir algo, no se necesita poder aplicarle las cuatro categorías para conocer algo.

Rosmini continúa, así, defendiendo su intuición básica: basta la idea del ser para ir percibiendo, incluso, intelectualmente las cosas. Por eso, subraya "Ciertamente, ninguna cosa corporal puede existir sin cantidad, cualidad y relación pero todas estas cosas que hay en ellas no le pertenecen, no tienen que ser percibidas intelectualmente por mí junto con ella para que sea posible decir que yo la he percibido o concebido" (2001:335). Para saber que algo existe o qué es basta con poder decir tal cosa es tal cosa; las personas comunes van por el mundo conociendo realidades sin pararse a pensar qué atributos tienen. Ésta es una interesante defensa del sentido común porque reconoce que se puede conocer sin tener que ir descubriendo todos los atributos de las cosas. Más aún, Rosmini reconoce que siempre hay muchas propiedades ocultas en las cosas que sólo con el tiempo y el estudio se podrán conocer, como también que normalmente las cosas podrían ser percibidas perfectamente sin ningún pensamiento acerca de estas propiedades o cualidades (2001:336). Este argumento no debe darnos la sensación de que basta con mirar y entender, pues, Rosmini reconoce que con tiempo y esfuerzo se va gradualmente perfeccionando el conocimiento. El conocimiento se da, entonces, por medio de un juicio de existencia o subsistencia de las propiedades de las cosas que se van añadiendo y que van haciendo, poco a poco, mejor nuestro conocimiento. El referirse y el atribuir estas propiedades a las cosas es posible, simplemente, porque conocer es descubrir las condiciones de existencia de las cosas no la posibilidad de su conocimiento.

\author{
Universidad de Tarapacá* \\ Departamento de Filosofía y Psicología \\ 18 de septiembre 2222 \\ Arica (Chile) \\ jalfonso@uta.cl
}




\section{BIBLIOGRAFÍA}

ALFONSO, Jorge. "El ser como principio: Unidad y Sistematicidad en la Filosofía de Antonio Rosmini". Pensamiento. Vol. 64, No 240. Madrid: Universidad de Comillas, 2008:251-266.

CASSIRER, Ernst. Kant, Vida y Doctrina. México: F. C. E., 1993.

FRANCK, Juan Francisco. "El problema del innatismo en Antonio Rosmini". II. Sapientia. Vol. LXII. Fasc. 221-222. Buenos Aires: Universidad Católica Argentina, 2007:53-76.

GIANNINI, Giorgio. La Metafísica de Antonio Rosmini. Córdoba: Convivio Filosófico, 1997.

------ "Il Divino e Dio in Rosmini". Scritti su Rosmini. Stresa: Rosminiane Sodalitas, 1994:9-24.

KANT, Immanuel. Crítica de la razón pura. Buenos Aires: Losada, 1961.

LÓPEZ-RIOBÓO, Blanca. La Crítica de Rosmini a la Gnoseología de Kant. Madrid: S. M., 1977.

MURATORE, Humberto. Antonio Rosmini. Vida y pensamiento. Madrid: BAC, 1998.

OTONELLO, Pier Paolo. "Il Divino e Dio", en Rosmini. L'ideale e il reale. Venezia: Marsilio, 1998:99-113.

------ La Ontologia di Rosmini. L'Aquila-Roma: JAPADRE, 1989.

ROSMINI, Antonio. A New Essay concerning the Origin of Ideas. Vols. 1, 2 \& 3. Durham: Rosmini House, 2001.

SCIACCA, Michelle Federico. I Principi Della Metafisica Rosminiana Opere III. 2. Palermo: L'Epos, 2003.

------ "Temas del pensamiento de Antonio Rosmini”, en Dianoia. México: UNAM, 1957:259-275. 